\title{
A HIGH-SPEED CAPACITIVE HUMIDITY SENSOR
}

\author{
Uksong Kang and Kensall D. Wise \\ Center for Integrated Sensors and Circuits \\ Department of Electrical Engineering and Computer Science \\ University of Michigan, Ann Arbor, MI 48109-2122
}

\begin{abstract}
This paper reports a high-speed capacitive humidity sensor with an integrated boron-diffused heater. A response time of $1.0 \mathrm{sec}$ and a sensitivity of $30.0 \mathrm{fF} / \% \mathrm{RH}$ have been obtained with the fabricated devices. High speed is achieved using multiple polyimide columns having diameters of a few microns and allowing moisture to diffuse into them circumferentially, altering the dielectric constant. A subsecond response time is expected when the geometry is further reduced. The purpose of the heater is to enable the sensor to recover from contamination and aging. It allows bake-out of the sensing film on demand and is useful in self-test protocols.
\end{abstract}

\section{INTRODUCTION}

Thin-film humidity sensors are widely used in many measurement and control applications, including those in automated process control, meteorology, domestic appliances, agriculture and medical equipment [1]. They can be categorized into different device types depending on the underlying sensing principle (capacitance, resistance, weight or stress changes in a thin film as a function of moisture absorption). Among these, capacitive devices are often preferred since they offer very low power consumption and a linear output response. Various ceramics, hygroscopic polymers, and electrolytes are used as humidity-sensitive materials.

In spite of a great deal of on-going research, thin-film humidity sensors continue to suffer from slow response $(>30 \mathrm{sec})$, low accuracy $(<+2 \% \mathrm{RH})$ and substantial long-term drift. Slow speed is of particular concern in applications involving transient humidity changes or measurements that must be taken on the fly, including many in industrial process control and in the monitoring of atmospheric relative humidity using radiosonds, dropsonds or unmanned aerial vehicles [2]. Contamination and aging of the sensing film is also an important issue since it causes the sensor to drift, resulting in decreased accuracy [3]. The sensing film can be reset by integrating a heater underneath the sensor.

\section{PRINCIPLE OF HIGH-SPEED HUMIDITY SENSING}

Capacitive humidity sensors detect moisture-induced changes in the dielectric constant of a hygroscopic polymer or ceramic layer to measure the ambient relative humidity level. In conventional structures, the diffusion of moisture into the sensing film takes place from only one side of the film as in Fig. 1(a). In contrast, as shown in Fig. 1(b), the high-speed structure used here absorbs moisture circumferentially into cylindrical islands. A significant improvement in speed is achieved.

In order to compare the response times of the conventional and the high-speed (circumferential) structures,

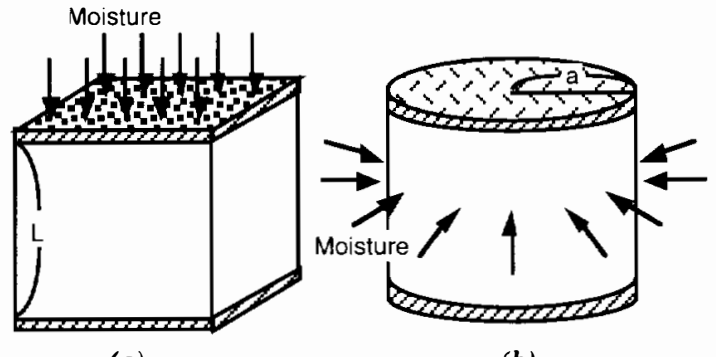

(a)

(b)

Figure 1. (a) Conventional structure (b) High-speed structure

the equations governing the transient capacitance change as moisture diffuses into a thin film or into a cylindrical body have been derive [4]. In this analysis, it is assumed that no moisture is present inside the film in its initial state and that the diffusion constant is independent of moisture concentration. The normalize capacitance change after an abrupt change in the ambient relative humidity level for single-sided diffusion into a rectangular body is formulated as

$$
C_{\text {Norm.rec }}(t)=\sum_{n=1}^{\infty} \frac{1-\exp \frac{-(2 n-1)^{2} \pi^{2} D t}{4 L^{2}}}{(2 n-1)^{2}} / \sum_{n=1}^{\infty} \frac{1}{(2 n-1)^{2}}
$$

where $t$ is the time, $D$ is the diffusion constant of moisture and $L$ is the height of the block. Similarly, the normalized capacitance for diffusion into a cylindrical body is derived as

$$
C_{\text {Normaty }}(t)=\sum_{n=1}^{\infty} \frac{1-\exp \left(-D k_{n}{ }^{2} t / a^{2}\right)}{k_{n}{ }^{2}} / \sum_{n=1}^{\infty} \frac{1}{k_{n}{ }^{2}}
$$

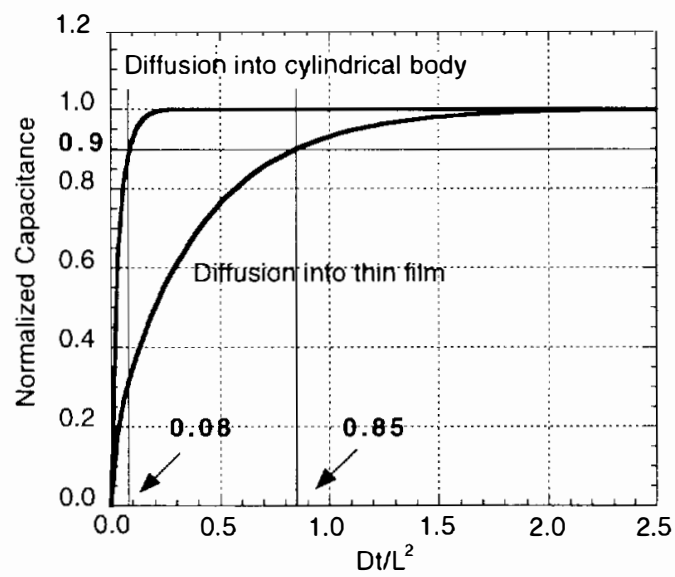

Figure 2. Analytical comparison of response times for diffusion into a thin film and into a cylindrical body. Improvement in the response time by a factor of 10 is obtained with the high-speed cylindrical structure. (t: time, $L$ : thickness of film $=$ diameter of cylinder, D: diffusion constant) 
where $a$ is the radius of the cylinder and the $k_{n}$ 's are roots of $J_{o}(k)=0 . \quad J_{o}$ is the zeroth-order Bessel function. Note that the normalized capacitance is a function of $D t / a^{2}$ from which it follows that the response time is proportional to the square of the radius and inversely proportional to the diffusion constant. Using equations (1) and (2), the normalized capacitance for both single-sided diffusion into a thin film and diffusion into a cylindrical body are plotted in Fig. 2. In this comparison, the hygroscopic film is of the same material for both cases and the thickness of the film is the same as the diameter of the cylinder. After an abrupt change in the ambient relative humidity level, it takes $0.85 L^{2} / D$ and $0.08 L^{2} / D$ seconds to reach the $90 \%$ point of the final steady state capacitance value for the conventional and the high-speed structure, respectively. Hence, an improvement in response time by about a factor of 10 is obtained with the high-speed structure.

\section{DEVICE STRUCTURE AND DESIGN}

A schematic diagram of the high-speed humidity sensor is shown in Fig. 3. The sensor is located on top of a boron-diffused heater and is electrically isolated from it by a thin dielectric layer. The sensor consists of thousands of polyimide columns, each of which has a diameter of a few microns. The columns are connected in parallel to form a capacitor. An upper electrode runs over the polyimide columns providing moisture free access to the sensing film. Besides serving as an electrode, this upper electrode also acts to filter out particles larger than the spacing between the adjacent conductors (a few microns). Contaminating gases can, in principle, be baked out of the polyimide using the integrated heater, enabling recovery of the sensing film from contamination, aging and hysteresis. A bulk-silicon borondiffused heater has been chosen to maintain planarity over the device surface. The device is mounted on a thermal insulator to increase the efficiency of the heater.

A preimidized, photosensitive polyimide (ULTRADEL 7501 - Amoco Chemicals) has been used as the moisture sensing material due to its thermal stability, mechanical robustness, and compatibility with VLSI process technology [5]. A preimidized material has been chosen to reduce stress build-up between the upper electrode and the polyimide. Preimidized polyimide shrinks in thickness by only about $8 \%$ after curing, while the polyimide precursor shrinks by as much as $50 \%$. Photosensitivity is necessary for forming the suspended topelectrode structure as will be described in the fabrication process sequence. ULTRADEL 7501 has a tensile modulus of

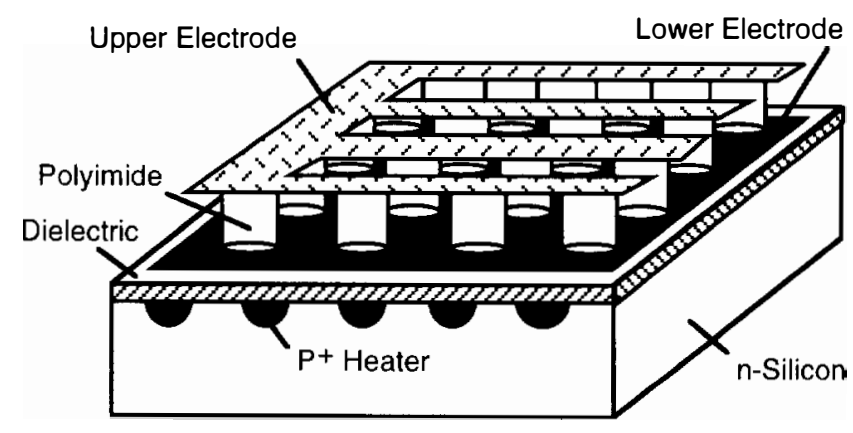

Figure 3. Schematic diagram of the high-speed capacitive humidity sensor with a boron-diffused heater.
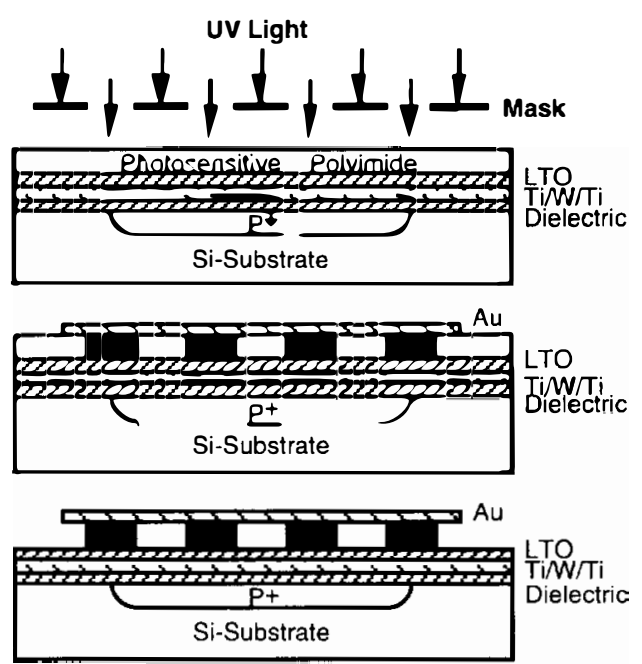

Figure 4. Fabrication process sequence for the high-speed capacitive humidity sensor.

510,000 psi, a thermal expansion coefficient at $200^{\circ} \mathrm{C}$ of $24 \mathrm{ppm} /{ }^{\circ} \mathrm{C}$, and a moisture uptake at $100 \% \mathrm{RH}$ of $3.4 \%$.

The capacitance of the sensor originates from both the polyimide and the air-gap. The polyimide capacitance is the one to be measured while the air-gap capacitance is a parasitic by-product of the electrical interconnections needed to access the capacitance of polyimide columns. Although the air-gap contributes to the total sensor capacitance it does not affect the sensitivity of the device since the dielectric constant of air changes by only about $1.4 \mathrm{ppm} / \% \mathrm{RH}$ while that of polyimide changes by $3330 \mathrm{ppm} / \% \mathrm{RH}$. The speed of the sensor is primarily determined by the diameter of polyimide columns. As noted above, the response time increases as the square of the column radius. The response time is not significantly influenced by the thickness of the polyimide film since diffusion takes place laterally. The sensitivity of the sensor for a given area, on the other hand, is determined by the thickness of the polyimide film as well as by its fill factor (the area that polyimide columns occupy per unit total area). The sensitivity becomes larger as the film thickness is reduced and the polyimide fill factor is increased.

\section{DEVICE FABRICATION}

The fabrication sequence is a 6 mask process. As shown in Fig. 4, fabrication starts with a deep boron diffusion into an n-type (100) wafer. A junction depth of $3 \mu \mathrm{m}$ forms the integrated heater. Then, a stress compensated LPCVD $\mathrm{SiO}_{2} /$ $\mathrm{Si}_{3} \mathrm{~N}_{4} / \mathrm{SiO}_{2}$ dielectric layer with a thickness of $0.5 \mu \mathrm{m} / 0.25 \mu \mathrm{m} /$ $0.5 \mu \mathrm{m}$ is deposited over the wafer. Contact holes to the heater are opened using RIE, followed by the deposition and lift-off of a Ti/W/Ti $(30 \mathrm{~nm} / 800 \mathrm{~nm} / 30 \mathrm{~nm})$ layer to act as the lower electrode. A $0.5 \mu \mathrm{m}$-thick low temperature oxide is now deposited over the wafer in order to eliminate the possibility of any conductive path between the upper and lower electrodes at high humidity or due to conductive particle trapping inside the air-gap. Vias are opened in the pad regions using BHF etching in order to enable wire bonding. After applying the adhesion promoter, a photosensitive polyimide (ULTRADEL 7501) is spun onto the wafer at $4500 \mathrm{rpm}$ for $60 \mathrm{sec}$, resulting in a film thickness of $2 \mu \mathrm{m}$. The polyimide columns are then defined 


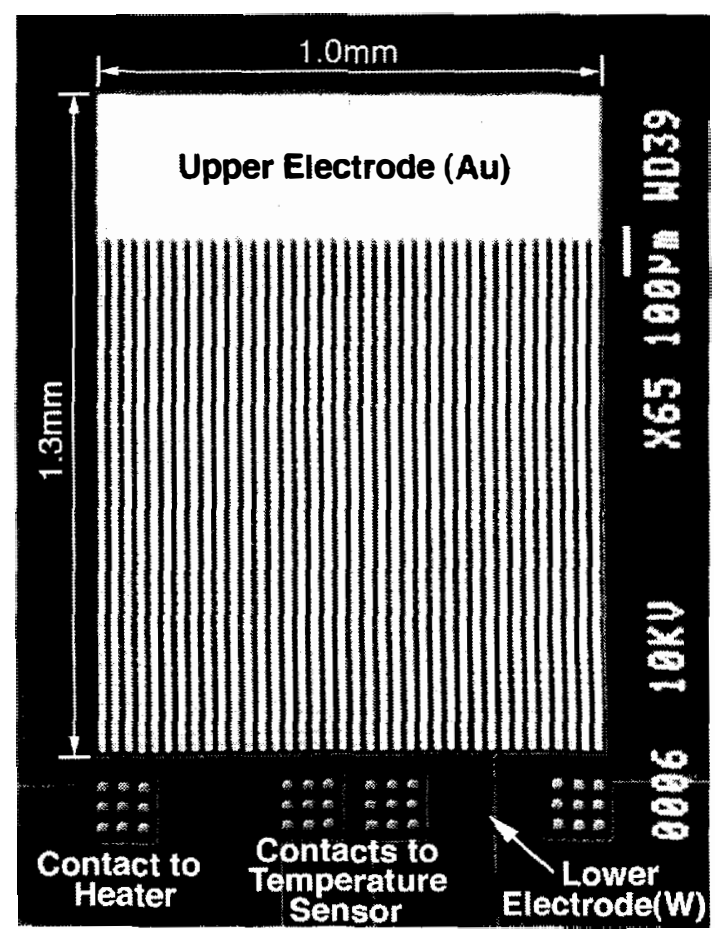

Figure 5. Top view of a fabricated type- $B$ device. The overall size of the sensor is $1.3 \mathrm{~mm} \times 1.0 \mathrm{~mm}$

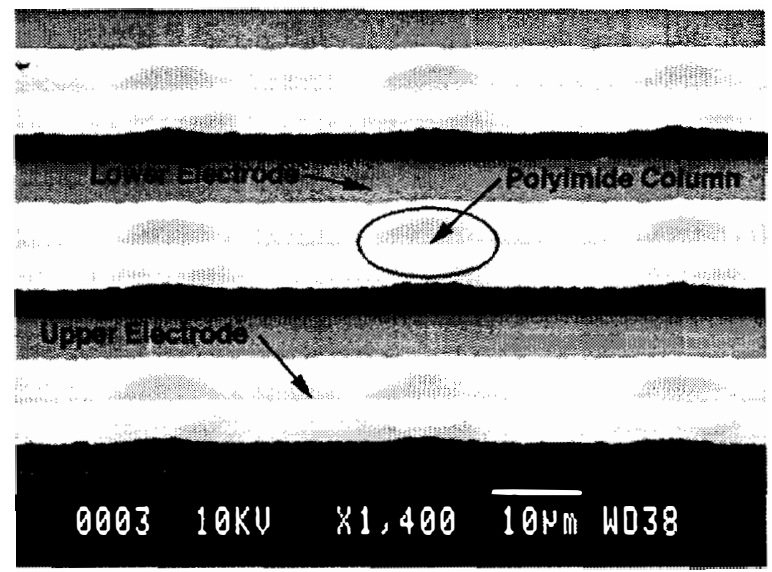

Figure 6. Close-up view of the upper electrode, lower electrode and polyimide columns.

lithographically followed by deposition of a Au layer with a thickness of $0.3 \mu \mathrm{m}$. The Au layer is patterned by wet etching to form the upper electrode. The polyimide is now developed so that the unexposed regions are dissolved away to leave the polyimide columns supporting the suspended upper electrode. Finally, the polyimide is cured in a nitrogen-purged oven at $300^{\circ} \mathrm{C}$ for 5 hours.

Three different sensors (types A, B and C) have been fabricated having polyimide column diameters of $5 \mu \mathrm{m}, 10 \mu \mathrm{m}$ and $15 \mu \mathrm{m}$, respectively. The total number of polyimide columns is 6138 for type A, 1900 for type B, and 891 for type C. An SEM picture of the type-B device is shown in Fig. 5. The overall size of the sensor is $1.3 \mathrm{~mm} \times 1.0 \mathrm{~mm}$. A serpentine boron-diffused heater and a boron-diffused temperature sensor are located underneath the sensor. The heater lines are $20 \mu \mathrm{m}$

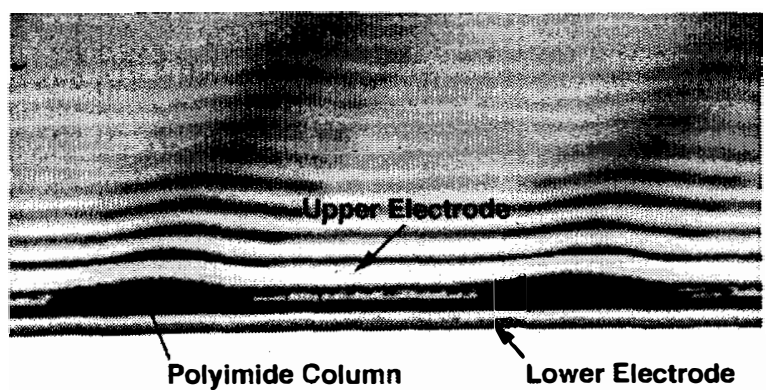

\section{$0004 \quad 10 \mathrm{KU} \quad X 2,300 \quad 10 \mathrm{Nm}$ W038}

Figure 7. Side-view of the upper electrode supported by polyimide columns under which there is the lower electrode. The thickness of the upper electrode is $0.3 \mu \mathrm{m}$ and that of polyimide is $2 \mu \mathrm{m}$.

wide and $3 \mu \mathrm{m}$ deep, and the measured heater resistance is $6.0 \mathrm{k} \Omega$. Contacts to the heater and temperature sensor are visible in the lower part of the picture. Electrical connection to the upper electrode is made directly to the Au rectangular pad by wire bonding. A close-up view of the upper metal strip lines supported by the polyimide columns is shown in Fig. 6, where the polyimide columns are visible as circles. A side-view is shown in Fig. 7.

\section{TEST RESULTS}

In Fig. 8, the measured output of the sensor is shown as a function of relative humidity at room temperature and a measurement frequency of $1 \mathrm{MHz}$. The sensor output is linear with a sensitivity of $30.6 \mathrm{fF} / \% \mathrm{RH}, \quad 25.8 \mathrm{fF} / \% \mathrm{RH}, \quad$ and $31.3 \mathrm{fF} / \% \mathrm{RH}$ for types $\mathrm{A}, \mathrm{B}$, and $\mathrm{C}$, respectively.

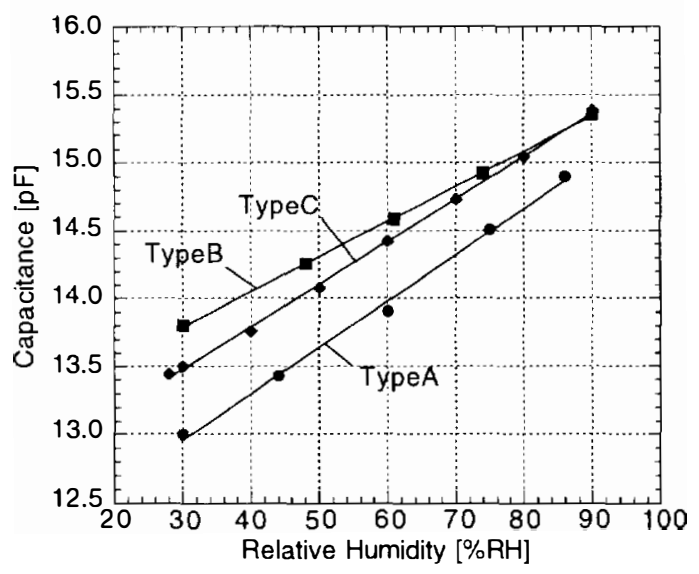

Figure 8. Sensor output as a function of relative humidity at room temperature for types $A, B$ and $C$ devices, which have polyimide column diameters of $5 \mu \mathrm{m}, 10 \mu \mathrm{m}$ and $15 \mu \mathrm{m}$, respectively.

The response times of the fabricated devices have been measured and are shown in Fig. 9. A significant improvement 
in speed has been obtained. The response times for types A, B and $C$ sensors are $1.0 \mathrm{sec}, 1.9 \mathrm{sec}$ and $6.9 \mathrm{sec}$, respectively. The response time is measured to the $90 \%$-point of the final steadystate capacitance value after an abrupt change in the ambient relative humidity level from $33 \% \mathrm{RH}$ to $90 \% \mathrm{RH}$.

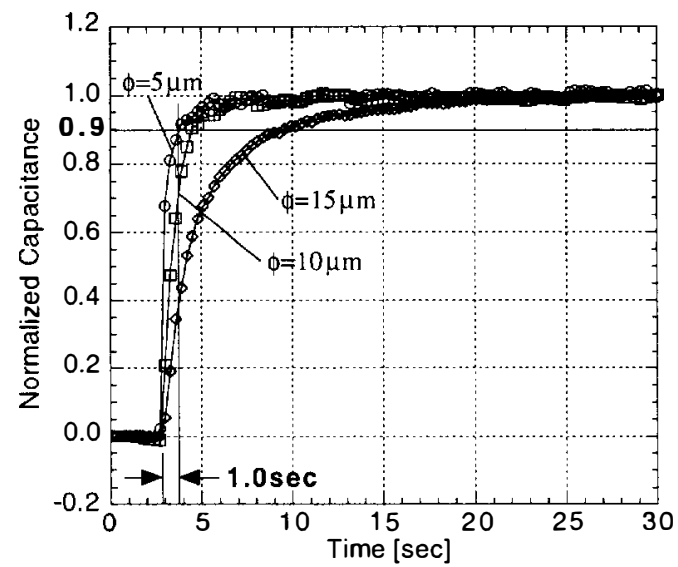

Figure 9. Response of sensors having polyimide column diameters of $5 \mu \mathrm{m}, 10 \mu \mathrm{m}$ and $15 \mu \mathrm{m}$ to an abrupt change in ambient relative humidity from $33 \% R H$ to $90 \% R H$ at room temperature.

The experimental set-up to expose the sensor to an abrupt change in relative humidity consists of a plastic container with a volume of $118 \mathrm{ml}$ located inside a temperaturehumidity chamber. The humidity sensor is mounted on a platform the end of which is attached with a hinge to the wall of the container. A spring fixed undemeath the platform enables the sensor to be transferred out of the container when the cap is opened. Two thirds of the container is filled with a saturated solution of $\mathrm{MgCl}_{2}$, which maintains the trapped air at $33 \% \mathrm{RH}$ when the container is sealed gas-tight and left for a sufficient amount of time. Using saturated salt solutions to calibrate humidity sensors is an inexpensive and practical method often used in industry [6]. After sealing the cap of the container with silicon rubber and waiting at least for 6 hours the chamber is set to $90 \% \mathrm{RH}$. When the chamber settles to this level, the cap is

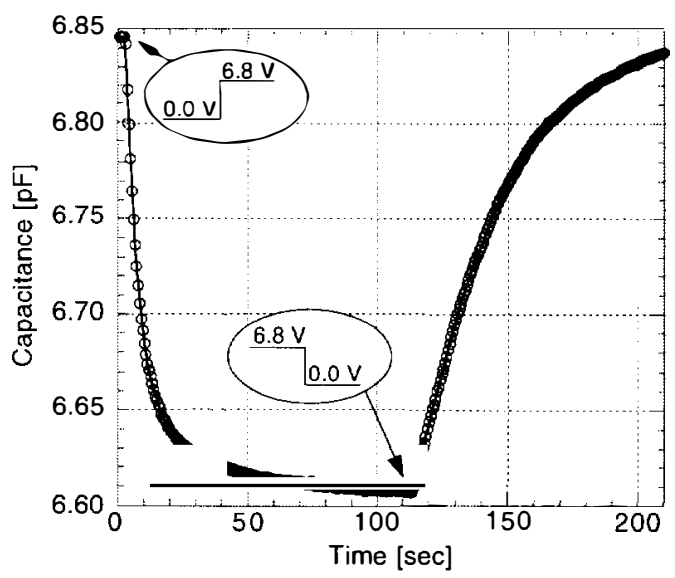

Figure 10. Response of the type-C device to a $6.8 \mathrm{~V}$ pulse applied to the heater $\left(60 \% R H, 25^{\circ} \mathrm{C}\right)$. opened by pulling a wire attached to the cap. The spring action causes the sensor to be transferred out of container, resulting in an abrupt change from $33 \% \mathrm{RH}$ to $90 \% \mathrm{RH}$. The container is positioned such that the sensor faces into air flow generated by a fan at the back of the chamber. The fan forces air at $90 \% \mathrm{RH}$ onto the sensor.

The sensor response to a $6.8 \mathrm{~V}$ pulse applied to the heater is shown in Fig. 10. A type $\mathrm{C}$ device has been used here with the ambient set at $60 \% \mathrm{RH}$ and $25^{\circ} \mathrm{C}$. As the supply voltage across the heater is increased from $0.0 \mathrm{~V}$ to $6.8 \mathrm{~V}$, the sensor capacitance decreases by about $250 \mathrm{fF}$. As the heater is turned off, the sensor capacitance returns to its original value. The temperature sensor indicates a temperature rise on the chip of about $20^{\circ} \mathrm{C}$ due to this excitation. This decrease in capacitance with heating is believed to be primarily due to baking out moisture in the film rather than to any thermal expansion.

\section{CONCLUSIONS}

A high-speed humidity sensor has been designed, fabricated and tested. Three different device types have been fabricated having polyimide column diameters of $5 \mu \mathrm{m}, 10 \mu \mathrm{m}$ and $15 \mu \mathrm{m}$. Response times of $1.0 \mathrm{sec}, 1.9 \mathrm{sec}$, and $6.9 \mathrm{sec}$ have been measured for these devices, respectively. A subsecond response time is expected if the diameter of the polyimide columns is further reduced. The corresponding device sensitivities are $30.6 \mathrm{fF} / \% \mathrm{RH}, 25.8 \mathrm{fF} / \% \mathrm{RH}$, and $31.3 \mathrm{fF} / \% \mathrm{RH}$. The sensor outputs are highly linear with relative humidity. The response of the humidity sensor to heating has also been tested. The sensor capacitance decreases by about $250 \mathrm{fF}$ as the device temperature is increased from $25^{\circ} \mathrm{C}$ to $45^{\circ} \mathrm{C}$ and moisture is baked out of the sensing film.

\section{ACKNOWLEDGMENTS}

The authors would like to gratefully acknowledge the support provided by the Defense Advanced Research Projects Agency under contract DABT63-95-C-0111.

\section{REFERENCES}

1. N. Yamazoe, "Humidity Sensors: Principles and Applications", Sensors and Actuators, 10, 379 (1986).

2. A. Mason, N. Yazdi, K. Najafi, and K. D. Wise, "A LowPower Wireless Microinstrumentation System for Environmental Monitoring", Proc. Transducers'95, Stockholm, Sweden, June (1995), pp. 107 - 110.

3. A.R.K. Ralston, M. C. Buncick and D. D. Denton, "Effects of Aging on Polyimide : a Model for Dielectric Behavior", Technical Digest of the 1990 Solid-State Sensors and Actuators Workshop, Hilton Head Isl., SC (1990), pp. 759 - 763.

4. J. Crank, The Mathematics of Diffusion, Chapter 5-6, Oxford University Press, London (1975).

5. T. Boltzhauser, L. Chandran, H. Baltes, and D. Steiner, "Humidity Sensing Properties and Electrical Permittivity of New Photosensitive Polyimides", Sensors and Actuators B, 5, 161 (1991).

6. A. Carotenuto, and M. Dell'Isola, "An Experimental Verification of Saturated Salt Solution-Based Humidity Fixed Points", International Journal of Thermophysics, 17, 1423 (1996). 